\title{
String theory and non-commutative gauge theory
}

\author{
Edward Witten \\ California Institute of Technology, Pasadena, CA 91106, USA \\ and \\ Institute for Advanced Study, Olden Lane, Princeton, NJ 08540, USA
}

Received 9 November 1999

Abstract. I describe some recent developments concerning the role of non-commutative YangMills theory in string theory.

PACS number: 1125

In this paper, I will report on work with N Seiberg [1] in which we attempted to understand in a more systematic framework the relation of string theory to non-commutative Yang-Mills theory. Such a relationship was first uncovered by Connes, Douglas and Schwarz in the context of matrix theories [2]. Two of the papers most relevant to this paper are that of Schomerus [3], attempting to extract a non-commutative parameter directly from conformal field theory, and that of Nekrasov and Schwarz on instantons on non-commutative $\mathbb{R}^{4}$ [4]. For additional references to the extensive literature, I refer to [1]. Further aspects of the subject have been explained by Seiberg [5].

The original application of non-commutative Yang-Mills theory to string theory [2] was to compactification on $\mathbb{T}^{2}$ (or $\mathbb{T}^{n}$ with $n>2$ ) in the limit of small area, with a fixed, non-zero value of

$$
\vartheta=\int_{\mathbb{T}^{2}} B .
$$

Here $B$ is the Neveu-Schwarz 2-form field that couples to the elementary string worldsheet $\Sigma$ via the familiar coupling $\int_{\Sigma} B$.

\section{Instantons}

At first sight, the discussion in [2] seemed to be quite tied to the small-area limit. Nekrasov and Schwarz [4] then gave a fascinating application of non-commutative Yang-Mills theory to string theory that did not involve small area at all. They considered string theory instantons on $\mathbb{R}^{4}$ in the presence of a constant, non-zero $B$-field, and claimed that they have the same moduli space as instantons on a non-commutative $\mathbb{R}^{4}$, with a non-commutativity parameter determined by $B$.

To be more specific, in flat $\mathbb{R}^{10}$ consider $N$ parallel 3-branes, with the worldvolume a flat $\mathbb{R}^{4}$ linearly embedded in $\mathbb{R}^{10}$. On the worldvolume of this system, there is a $U(N)$ gauge symmetry, with unbroken four-dimensional $\mathcal{N}=4$ supersymmetry. Now introduce a constant $B$-field, that is a $B$-field with constant components $B_{I J}, I, J=1, \ldots, 4$. Note that in the 
presence of the branes, a constant $B$-field cannot be gauged away (as this would generate a constant magnetic field $F$ on the branes; to describe the situation more accurately, there is a gauge-invariant combination $F+B$ that cannot be gauged away).

Now, let us look at gauge configurations on the branes that preserve half of the supersymmetry. In the field theory limit, these are Yang-Mills instantons, that is, solutions of the instanton equation $F_{I J}^{+}=0$ (here $F^{+}$is the self-dual projection of $F$ ). The classical instanton equation is scale-invariant, and classical instantons come in all sizes.

Going over to string theory, for sufficiently big instantons, the classical instanton equation is in fact a good approximation. As the instanton shrinks, we generically expect that $\alpha^{\prime}$ corrections will become important. However, as explained in section 2.2 of [1], with a suitable regularization there are in fact no $\alpha^{\prime}$ corrections to the instanton moduli space if $B=0$. One shows this by writing down the sigma model for open strings ending on the 3-branes with boundary coupling to gauge fields, with a certain kind of Pauli-Villars regularization. Spacetime supersymmetry is equivalent to the existence of a certain $S U(2)$ group of $R$ symmetries, and this condition gives the classical instanton equation. For further details on this argument, consult [1].

In particular, it follows from this that at $B=0$, the stringy instanton moduli space is the same as the classical one, and thus there is a small-instanton singularity, the singularity that appears in the moduli space when an instanton shrinks to zero size. Indeed, the meaning of the small-instanton singularity in string theory is familiar. An instanton can shrink to zero size and escape as a -1-brane; there is a singularity where the two branches (instantons and -1-branes) meet.

Now, we turn to $B \neq 0$. We find that if $B^{+} \neq 0$, there can be no small-instanton singularity since a state consisting of a 3-brane and a separated -1-brane is not supersymmetric. There is an energetic barrier separating the -1-brane from the 3-branes, and hence the usual smallinstanton singularity must be absent for $B^{+} \neq 0$.

So the instanton moduli space must have $\alpha^{\prime}$ corrections at $B^{+} \neq 0$. Indeed, Nekrasov and Schwarz proposed that at $B \neq 0$, the moduli space of stringy instantons is equal to the moduli space of 'instantons on a non-commutative $\mathbb{R}^{4}$.

What are they? For this, we must recall the definition of non-commutative Yang-Mills theory [6]. We start with a Poisson bracket of functions on $\mathbb{R}^{4}$ :

$$
\{f, g\}=\theta^{i j} \partial_{i} f \partial_{j} g .
$$

Here $\theta$ is a bivector with constant coefficients $\theta^{i j}$; it will be determined in terms of $B$. Now deform the $f$ 's to elements $\widehat{f}$ of a non-commutative associative algebra $\mathcal{A}$ with multiplication denoted by $*$ and

$$
\widehat{f} * \widehat{g}-\widehat{g} * \widehat{f}=\mathrm{i}\{f, g\}+\mathcal{O}\left(\theta^{2}\right) .
$$

(One further requires that the $*$ product has an expansion in powers of $\theta$, with each term given by a local expression, of finite order in derivatives of $f$ and $g$.) There is an essentially unique such product, up to a certain equivalence relation. It can be written explicitly as

$$
f * g(x)=\left.\exp \left(\frac{\mathrm{i}}{2} \theta^{i j} \frac{\partial}{\partial y^{i}} \frac{\partial}{\partial z^{j}}\right) f(y) g(z)\right|_{y=z=x} .
$$

This formula defines what is known as the Moyal bracket of functions on $\mathbb{R}^{4}$.

Once the algebra $\mathcal{A}$ has been defined, one imitates the familiar definitions in gauge theory. We consider first the rank-1 case. The gauge parameter $\widehat{\lambda}$ is a function, that is, an element of $\mathcal{A}$. The components $\widehat{A_{i}}$ of the gauge field are likewise elements of $\mathcal{A}$. The gauge transformation law is

$$
\delta \widehat{A}_{i}=\partial_{i} \widehat{\lambda}_{i}+\mathrm{i} \widehat{\lambda} * \widehat{A}_{i}-\mathrm{i} \widehat{A}_{i} * \widehat{\lambda}
$$


just like in Yang-Mills theory, but with $*$ products instead of matrix multiplication. The gauge-covariant field strength is likewise

with

$$
\widehat{F}_{i j}=\partial_{i} \widehat{A}_{j}-\partial_{j} \widehat{A}_{i}+\mathrm{i} \widehat{A}_{i} * \widehat{A}_{j}-\mathrm{i} \widehat{A}_{j} * \widehat{A}_{i},
$$

$$
\delta \widehat{F}_{i j}=\mathrm{i} \widehat{\lambda} * \widehat{F}_{i j}-\mathrm{i} \widehat{F}_{i j} * \widehat{\lambda} .
$$

For the rank- $N$ version, one simply replaces $\mathcal{A}$ by its tensor product with the algebra of $N \times N$ complex matrices. Then, reinterpreting $*$ as the tensor product of the $*$ product as defined above with matrix multiplication, one uses the above formulae.

Now, we can define what we mean by an instanton on a non-commutative $\mathbb{R}^{4}$. It is simply a non-commutative gauge field, in the above sense, for which the non-commutative field strength $\widehat{F}$ obeys $\widehat{F}^{+}=0$. Nekrasov and Schwarz showed that the moduli space $\widehat{\mathcal{M}}$ of such objects is given by a deformation of the ADHM equations to include a 'Fayet-Iliopoulos' additive constant. This deformation has been studied mathematically [7] as a partial desingularization of the classical instanton moduli space $\mathcal{M}$, and also arises [8] in matrix theory in the presence of a $B$-field.

It turns out that the stringy instanton moduli space is indeed the non-commutative instanton moduli space $\widehat{\mathcal{M}}$, whenever $B^{+} \neq 0$. A detailed discussion is given in [1], and we add a few words below. However, jumping ahead of our story a bit, non-commutative Yang-Mills is most effective in describing the physics for very large $B$, i.e. $\alpha^{\prime}|B| \gg 1$. Note that $|B| \rightarrow \infty$ is part of the story of Connes et al [2], since they took the area of a 2-torus $\mathbb{T}^{2}$ (on which the theory was compactified) to zero with fixed $\int_{\mathbb{T}^{2}} B$; this clearly implies that $|B| \rightarrow \infty$. ( $|B|$ is defined as $\sqrt{g^{I K} g^{J L} B_{I J} B_{K L}}$.) So the $|B| \rightarrow \infty$ limit is quite close to the starting point of the subject.

Also, we should not leave the subject of instantons without noting that there is something very strange about describing stringy instantons via solutions of a non-commutative instanton equation $\widehat{F}^{+}=0$. If this is so for instantons in general, it must be true in particular for big instantons, with a scale size much bigger than the string scale. However, it should be possible, even for non-zero $B$, to describe big instantons using ordinary gauge fields, obeying an equation that differs from the classical instanton equations by $\alpha^{\prime}$ corrections (which can be computed via sigma models, for instance) that preserve the ordinary Yang-Mills gauge invariance. So a simple equation $\widehat{F}^{+}=0$ with non-commutative gauge invariance must be equivalent to a more complicated equation with ordinary gauge invariance. Before explaining this rather surprising fact, we will restate the problem in a more general context.

\section{Open strings in a magnetic field}

I have introduced our discussion of the role of non-commutative Yang-Mills theory by considering instantons, because they give a very striking application, where string theory is highly non-classical but can be described in great detail. This contrasts, say, with Calabi-Yau theory, where a full description is way out of reach. However, now we are going to leave the instantons behind. For the rest of this paper, we will try to derive non-commutative Yang-Mills systematically from open strings in a constant magnetic field, a problem studied extensively in the mid-1980s $[9,10]$. In the bosonic case, the worldsheet action is

$$
I=\frac{1}{4 \pi \alpha^{\prime}} \int \mathrm{d}^{2} z g_{I J} \partial_{z} X^{I} \partial_{\bar{z}} X^{J}-\frac{\mathrm{i}}{2} \int B_{I J} \mathrm{~d} X^{I} \wedge \mathrm{d} X^{J} .
$$

We will be considering open string vertex operators only. To evaluate their correlators, we need the propagator

$$
\left\langle X^{I}(\tau) X^{J}\left(\tau^{\prime}\right)\right\rangle
$$


for $\tau, \tau^{\prime}$ on the boundary. This was found long ago to be

$$
\left\langle X^{I}(\tau) X^{J}\left(\tau^{\prime}\right)\right\rangle=-\alpha^{\prime} G^{I J} \ln \left(\tau-\tau^{\prime}\right)^{2}+\mathrm{i} \frac{1}{2} \theta^{I J} \epsilon\left(\tau-\tau^{\prime}\right),
$$

where

$$
G^{I J}=\left(\frac{1}{g+2 \pi \alpha^{\prime} B}\right)_{S Y M}^{I J}
$$

and

$$
\theta^{I J}=2 \pi \alpha^{\prime}\left(\frac{1}{g+2 \pi \alpha^{\prime} B}\right)_{A N T I}^{I J} .
$$

Here the subscripts $S Y M$ and $A N T I$ are instructions to take the symmetric or antisymmetric part of a matrix.

The logarithmic term in the propagator determines the anomalous dimensions of operators. For instance, the tachyon vertex operator $\exp (\mathrm{i} p \cdot X)$ has dimension $\frac{1}{2} \alpha^{\prime} G^{I J} p_{I} p_{J}$, so $G^{I J}$ (or rather its inverse $G_{I J}$ ) is the effective metric seen by the open strings. On the other hand, as suggested in [3], $\theta$ determines the non-commutativity. We have indeed for $\tau \rightarrow \tau^{\prime}$ with $\tau>\tau^{\prime}$

$$
\mathrm{e}^{\mathrm{i} p \cdot X}(\tau) \mathrm{e}^{\mathrm{i} q \cdot X}\left(\tau^{\prime}\right) \rightarrow\left|\tau-\tau^{\prime}\right|^{\alpha^{\prime} G^{I J} p_{I} q_{J}} \exp \left(\frac{1}{2} \mathrm{i} \theta^{I J} p_{I} q_{J}\right) \mathrm{e}^{\mathrm{i}(p+q) \cdot X}\left(\tau^{\prime}\right) .
$$

If we could ignore the factor $\left|\tau-\tau^{\prime}\right|^{\alpha^{\prime} G^{I J} p_{I} q_{J}}$, we would recognize the $*$ product

$$
\mathrm{e}^{\mathrm{i} p \cdot X} * \mathrm{e}^{\mathrm{i} q \cdot X}=\exp \left(\frac{1}{2} \mathrm{i} \theta^{I J} p_{I} q_{J}\right) \mathrm{e}^{\mathrm{i}(p+q) \cdot X} .
$$

Of course, it is completely wrong in general to ignore this factor. It is closely tied up with the usual anomalous dimensions of operators and the whole standard worldsheet structure. String theory just would not work without it.

The rest of this paper has two parts:

(a) What we can say in general.

(b) What we can say when we get rid of the anomalous dimensions.

\section{General B dependence of the effective action}

Note first that the term in the propagator involving $\theta$ is a piecewise-constant function of $\tau$ and $\tau^{\prime}$, and hence does not contribute to correlation functions of $\mathrm{d} X / \mathrm{d} \tau$ or of higher derivatives $\mathrm{d}^{m} X / \mathrm{d} \tau^{m}, m>1$. Suppose now that we consider open strings with $U(N)$ Chan-Paton factors. Consider the tree-level scattering of $k$ gauge bosons of momenta $p_{i}$, polarizations $\epsilon_{i}$ and ChanPaton wavefunctions $\lambda_{i}$, with $i=1, \ldots, k$. Tree level means that the worldsheet is a disc. The scattering amplitude is

$$
A\left(\lambda_{i}, \epsilon_{i}, p_{i}\right)_{G, \theta}=\operatorname{Tr} \lambda_{1} \lambda_{2} \ldots \lambda_{k} \int \mathrm{d} \tau_{i}^{\prime}\left\langle\prod_{i=1}^{k} \epsilon_{i} \cdot \frac{\mathrm{d} X}{\mathrm{~d} \tau} \mathrm{e}^{\mathrm{i} p_{i} \cdot X}\left(\tau_{i}\right)\right\rangle_{G, \theta} .
$$

The subscripts $G$ and $\theta$ mean that the amplitudes and correlation functions are evaluated as a function of $G$ and $\theta$, respectively. The vertex operators are inserted on the boundary of the disc in a definite cyclic order; the symbol $\mathrm{d} \tau_{i}^{\prime}$ refers to an integral over the positions of the $\tau_{i}$ modulo the action of $S L(2, \mathbb{R})$. The only $\theta$ dependence of the correlation function that appears in the above formula for the amplitude is a factor

$$
\exp \left(-\frac{1}{2} \mathrm{i} \sum_{s>r} \theta^{I J} p_{s, I} p_{r, J}\right)
$$

that comes from the expectation value of the product of exponential operators. This factor, which arises in evaluating the correlation function that appears inside the $d \tau_{i}^{\prime}$ integral, is a 
piecewise-constant function of the $\tau_{i}$ that depends only on their cyclic ordering; this cyclic ordering is kept fixed in evaluating the integral that gives the scattering amplitude. Hence, this factor appears as an overall factor multiplying an otherwise $\theta$-independent amplitude.

Given the tree-level $S$-matrix, one can, at $\theta=0$, find a local effective action that generates it to any desired order in $\alpha^{\prime}$. It takes the general form

$$
S_{G}=\frac{1}{g_{s t}} \int \mathrm{d}^{n} x \sqrt{G} \operatorname{Tr}\left(G^{I K} G^{J L} F_{I J} F_{K L}+\alpha^{\prime} \text { corrections }\right) .
$$

The effective action is written as the spacetime integral of a single trace of a product of gaugecovariant fields (rather than the integral of a product of traces) since the scattering amplitude is proportional to a single trace $\operatorname{Tr} \lambda_{1} \lambda_{2} \ldots \lambda_{k}$ of Chan-Paton wavefunctions multiplied in cyclic order. We write the effective action as $S_{G}$ to emphasize that it depends on the metric $G$.

Now we want to incorporate the effects of having $\theta \neq 0$. For this, we must incorporate the phase factor in the scattering amplitude. This factor has the effect of replacing ordinary multiplication of wavefunctions by the $*$ product. This has the effect, for example, of replacing the standard definition of $F_{I J}$ by the definition we gave above of $\widehat{F}_{I J}$. The $\theta$ - and $G$-dependent effective action $S_{G, \theta}$ is obtained from $S_{G}$ by replacing everywhere $F$ by $\widehat{F}$ and replacing the matrix multiplication by $\mathrm{a} *$ product. Thus

$$
S_{G, \theta}=\frac{1}{g_{s t}} \int \mathrm{d}^{n} x \sqrt{G}\left(G^{I K} G^{J L} \widehat{F}_{I J} \widehat{F}_{K L}+\alpha^{\prime} \text { corrections }\right),
$$

with the same formula as at $\theta=0$, except that now everything is 'non-commutative'.

So, non-commutative Yang-Mills theory can be used to give a simple description of the $\theta$ or $B$ dependence of the effective action, to all orders in $\alpha^{\prime}$. (This also seems to imply that at $\theta \neq 0, \widehat{F}^{+}=0$ must be the exact instanton equation, given that at $\theta=0, F^{+}=0$ is exact. For if at $\theta=0$, the $F^{+}$dependence of the action is quadratic and higher order near an arbitrary solution of $F^{+}=0$, then at $\theta \neq 0$, the same will hold for the $\widehat{F}^{+}$dependence near an arbitrary solution of $\widehat{F}^{+}=0$.) In this non-commutative description, the effective action has a very complicated $\alpha^{\prime}$ expansion: the same as the $\alpha^{\prime}$ expansion one gets in the usual commutative description at $\theta=0$.

\section{Comparison with ordinary Yang-Mills theory}

On the other hand, by standard methods, such as a $\sigma$-model approach to the effective action using Pauli-Villars regularization, one can describe the effective action via local gaugeinvariant interactions with conventional Yang-Mills gauge invariance, to all orders in $\theta$ and $\alpha^{\prime}$. In this framework, the action is naturally written in terms of the 'original' or closed string metric $g$ which appeared in the worldsheet action, and the $B$ dependence is expressed by replacing $F$ with $F+B$.

Here is a familiar example: the case of $U(1)$ gauge fields with possibly large, but almost constant, field strength. The effective action is the Dirac-Born-Infeld action

$$
S=\frac{1}{g_{s t}} \int \mathrm{d}^{n} x \sqrt{\operatorname{det}\left(g+\alpha^{\prime} F+\alpha^{\prime} B\right)} .
$$

This action is expressed in terms of standard gauge-invariant interactions for an ordinary gauge field $A$, with conventional field strength $F$. It depends on both $\theta$ and $G$ (or $g$ and $B$ ) explicitly, not just via a $*$ product.

This seems like a contradiction. The same effective action for open string scattering can be described both by non-commutative Yang-Mills fields and also by standard Yang-Mills theory! The two frameworks must therefore be equivalent (by a transformation that changes 
the form of the action). There is, in fact, a completely explicit transformation that does this, to first order in $\theta$ (see [1] for details). To all orders in $\theta$, the transformation is generated by an explicit differential equation. An interesting feature of this transformation is that is does not map the gauge group of ordinary Yang-Mills to the gauge group of non-commutative YangMills (this would be impossible, since for instance in the rank-1 case one group is Abelian and one is non-Abelian). Rather, the transformation maps the gauge equivalence relation of one theory to the gauge equivalence relation of the other. This is good enough.

$\alpha^{\prime} \rightarrow 0$ limit

So far, our path to seeing non-commutative Yang-Mills in open string physics has gone via the $S$-matrix. It would be much nicer to extract the $*$ product, or a more general associative product including excited strings, directly from the OPEs of the worldsheet conformal field theory.

As we have seen above, the obstruction to doing this is in the anomalous dimensions of the operators. Indeed, if the dimensions of vertex operators vanished, we could obtain an associative but, in general, not commutative algebra simply from multiplication of vertex operators. If $V$ and $V^{\prime}$ are two vertex operators, we would define the product $V * V^{\prime}$ simply as $\lim _{\tau \rightarrow \tau^{\prime}} V(\tau) V^{\prime}\left(\tau^{\prime}\right)$. This would automatically be associative, since open string vertex operators are inserted on the boundary in a definite cyclic order, but in general it would not be commutative.

The dimensions of vertex operators spoil this naive definition. The limiting operator product $\lim _{\tau \rightarrow \tau^{\prime}} V(\tau) V^{\prime}\left(\tau^{\prime}\right)$ generically needs some renormalization when the dimensions do not vanish, and after making this renormalization to define $V * V^{\prime}$, one is unable to prove the associative relation $\left(V * V^{\prime}\right) * V^{\prime \prime}=V *\left(V^{\prime} * V^{\prime \prime}\right)$. In trying to prove this relation by considering a product of three vertex operators $V(\tau) V^{\prime}\left(\tau^{\prime}\right) V^{\prime \prime}\left(\tau^{\prime \prime}\right)$ with $\tau>\tau^{\prime}>\tau^{\prime \prime}$, one runs into the fact that the renormalization needed to define $\left(V * V^{\prime}\right) * V^{\prime \prime}$ from this operator product is different from the renormalization needed to define $V *\left(V^{\prime} * V^{\prime \prime}\right)$.

Precisely for this reason, in trying in the mid-1980s to base open string field theory on an associative algebra and on Connes's axioms for non-commutative geometry, it was necessary to use, not the operator product algebra directly, but a messy algebra defined in terms of gluing of strings [11].

Likewise, the anomalous dimensions prevent one from seeing either ordinary Yang-Mills gauge invariance (at $\theta=0$ ) or non-commutative Yang-Mills gauge invariance (for $\theta \neq 0$ ) directly from OPEs. At $\theta=0$, a standard way to make the gauge invariance manifest involves going to long wavelength, or equivalently, taking $\alpha^{\prime} \rightarrow 0$ at fixed wavelength. Then one makes a sigma-model expansion in powers of the propagator, which is of order $\alpha^{\prime}$. In this expansion, the anomalous dimensions vanish in the leading order, and in perturbing around this limit, we can make the gauge invariance manifest.

At $\theta \neq 0$, we can do exactly the same thing. We recall that our propagator is $-\alpha^{\prime} G^{I J} \ln \left(\tau-\tau^{\prime}\right)^{2}+\theta^{I J} \epsilon\left(\tau-\tau^{\prime}\right)$. To eliminate the anomalous dimensions while retaining the non-commutativity and obtaining a limit for the effective geometry seen by the open strings, we want to take $\alpha^{\prime} \rightarrow 0$ with fixed $G$ and $\theta$. Looking back to the formulae given earlier for defining $G$ and $\theta$ in terms of $g, B$ and $\alpha^{\prime}$, this can be done by taking $\alpha^{\prime}$ to zero, with $B$ fixed as a 2-form and $g$ also going to zero. Indeed, one can take $\alpha^{\prime} \sim \epsilon, g_{I J} \sim \epsilon^{1 / 2}, B_{I J} \sim 1$. (I am assuming that $B_{I J}$ is non-degenerate; otherwise, one makes this scaling of $g$ only in a subspace in which $B_{I J}$ is non-degenerate, and leaves $g$ fixed on the nullspace of $B$.) 
In this limit, the worldsheet action reduces to the interaction with the $B$-field:

$$
-\mathrm{i} \frac{1}{2} \int_{\Sigma} B_{I J} \mathrm{~d} X^{I} \wedge \mathrm{d} X^{J}
$$

This is invariant under diffeomorphisms of the worldsheet $\Sigma$, which perhaps is the basic reason that this limit is so simple. Since we have taken $\alpha^{\prime} \rightarrow 0$, the spacetime action reduces to

$$
S_{G, \theta}=\frac{1}{g_{s t}} \int \mathrm{d}^{n} x \sqrt{G} G^{I K} G^{J L} \operatorname{Tr}\left(\widehat{F}_{I J} \widehat{F}_{K L}\right),
$$

with vanishing of the $\alpha^{\prime}$ corrections.

Keeping $B_{I J}$ fixed while scaling $g_{I J}$ to zero gives us, if we are on a torus, the small-volume limit with fixed periods of $B$. This is the limit where the relevance of non-commutative YangMills theory to physics was argued in [2]. Thus we have returned more or less to the starting point, having hopefully learned something new along the way.

\section{Acknowledgments}

This work was supported in part by NSF Grant PHY-9513835 and the Caltech Discovery Fund.

\section{References}

[1] Seiberg N and Witten E 1999 J. High Energy Phys. JHEP09(1999)032

(Seiberg N and Witten E 1999 String theory and noncommutative geometry Preprint hep-th/9908142)

[2] Connes A, Douglas M and Schwarz A 1998 Noncommutative geometry and matrix theory: compactification on tori J. High Energy Phys. JHEP02(1998)003

(Connes A, Douglas M and Schwarz A 1997 Preprint hep-th/9711162)

[3] Schomerus V 1999 D-branes and deformation quantization J. High Energy Phys. JHEP06(1999)030 (Schomerus V 1999 Preprint hep-th/9903205)

[4] Nekrasov N and Schwarz A 1998 Instantons on noncommutative $\mathbf{R}^{4}$ and $(2,0)$ superconformal field theory Commun. Math. Phys. 198689

(Nekrasov N and Schwarz A 1998 Preprint hep-th/9802068)

[5] Seiberg N 1999 Talk presented at Strings '99, Potsdam, 19-24 July 1999 (available at http:strings99.aeipotsdam.mpg.de/cgi-bin/viewit.cgi?speaker=Seiberg)

[6] Connes A and Rieffel M 1987 Yang-Mills for noncommutative two-tori Operator Algebras and Mathematical Physics (Iowa City, IA, 1985) (Contemp. Math. Oper. Alg. Math. Phys. vol 62) (Providence, RI: American Mathematical Society) p 237

[7] Nakajima H 1994 Resolutions of moduli spaces of ideal instantons on $\mathbf{R}^{4}$ Topology, Geometry and Field Theory ed K Fukuya, M Furuta, T Kohno and D Kotschick (Singapore: World Scientific)

[8] Aharony O, Berkooz M and Seiberg N 1995 Light cone description of $(2,0)$ superconformal theories in six dimensions Adv. Theor. Math. Phys. 2541

(Aharony O, Berkooz M and Seiberg N 1995 Preprint hep-th/9511030)

[9] Fradkin E S and Tseytlin A A 1985 Nonlinear electrodynamics from quantized strings Phys. Lett. B 163123

[10] Callan C G Jr, Lovelace C, Nappi C R and Yost S A 1987 Open strings in background gauge fields Nucl. Phys. B 280599

[11] Witten E 1986 Noncommutative geometry and string field theory Nucl. Phys. B 268253 